\title{
An Insight into Software Crowdsourcing: How Crowd can transform the Business Model for Technology Service Providers
}

\author{
Dhananjay Mehta \\ Software Engineer \\ Accenture Services Pvt. Ltd. \\ Pune, India
}

\begin{abstract}
Crowdsourcing is a distributed computing model which enables sharing of human efforts in form of ideas, nonhierarchical decision making; utilization of human intelligence and collaboration on complex real world problems that otherwise cannot be solved by any individual person , machine or organization easily. It leverage machine learning, automation, artificial intelligence and collective intelligence into real world challenges combining computation, mobile devices and social interaction to achieve large-scale distributed computing. This paper examines how software crowdsourcing can be helpful for technology service providers like IBM, TCS, Infosys, Accenture etc. and its potentials in transforming present technology outsourcing business model.
\end{abstract}

\section{Keywords}

Crowdcomputing, Crowdsourcing, Collective Intelligence, Enterprise Crowdsourcing.

\section{INTRODUCTION}

Crowd sourcing is a distributed problem solving model which has emerged in recent years. The term crowdsourcing was coined by Jeff Howe and Mark Robinson in the June 2006 issue of Wired magazine [1]. Crowdsourcing tends to harness the efforts of individuals over a distributed network. It aims to narrow the disparity between the understanding and computation power of humans and computers. There is no well-defined definition for crowdsourcing and it can be looked from various perspectives be it problem resolution or innovation [2]. Many crowdsourcing platforms have been built today e.g. Mechanical Turk (Amazon), Turkit, Mob4hire, oDesk, Topcoder, 99design, Inno-centive, Cloud Crowd etc. With more and more technically skilled people having access to internet today, crowdsourcing provide a pool of highly skilled and cheap resources.

\section{RESEARCH DESIGN}

Firstly this paper introduces software crowdsourcing and illustrates some crowdsourcing initiatives. It then focuses on present outsourcing model and highlights the need of crowdsourcing. In the later sections it describes enterprise crowdsourcing and potentials it holds for outsourcing business model. Towards the end, the paper proposes design of a basic software crowdsourcing platform able to overcome present challenges.

\section{WHAT IS CROWDSOURCING?}

Crowdsourcing is a compound contraction of 'Crowd' and 'Outsourcing' means outsourcing to crowd [3].In Howe's words crowdsourcing is an act of taking tasks once performed by the employees to an anonymous and undefined network of people or "crowd". In simpler words, crowdsourcing is outsourcing a piece of work to a large number of internet connected, location independent, volatile workforce that collaborate small pieces of work to provide an overall outcome.

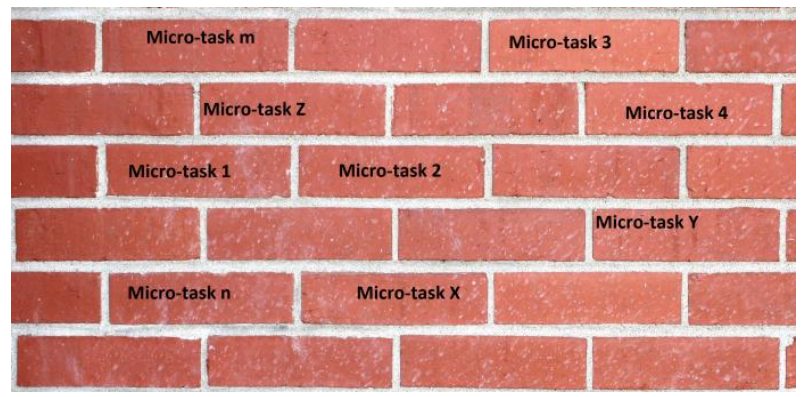

Fig 1: Analogy between brick wall and crowdsourcing.

This analogy is similar to a brick wall, where each brick (micro task) is laid down to create a wall (complex task). In this section we will study the elements of crowd system and illustrate this initiative through examples.

\subsection{Elements of a Crowd System}

Defining distinctive boundaries of a crowdsourced system is difficult as it has evolved over a long period of time. The very foundation to crowd sourcing was laid in 1970-80s by the open-source initiatives which saw people across the globe collaborating to work over projects. This was followed by outsourcing in 1980s where the tasks performed by an organization were divided and collectively performed by different vendors. Toward the end of the 20th century 'freelancing' came into picture where micro tasks were performed by individuals. With advancement of computation technologies over this decade crowdsourcing has come into picture where a crowd explicitly collaborates to build a large complex system. Crowdsourcing is very different from other initiatives such as Wikipedia, Linux or other such initiatives where individuals work collaboratively. Crowdsourcing is organization sponsored process and involve a "crowd" which is directed or managed by this organization throughout the process. Open source projects are participatory whereas crowdsourcing rely on collective intelligence [4]. Crowdsourcing involves three elements [3]:

1. Organization offering tasks to be performed.

2. Individuals forming the crowd and performing the tasks.

3. A crowdsourcing platform to perform and integrate the micro tasks. 
These are basic elements of a crude crowd system; advanced systems may also include Artificial Intelligence, Integration tools, Automation Tools, Workflow design and management, user access and control management etc. [11]. The crowd can be classified in two categories: Public Crowd which comprise of anonymous group of individuals who randomly collaborate on projects and Private Crowd consisting of known group of individuals who collaborate together over tasks and projects regularly.

\subsection{Software Crowdsourcing}

Any software development process involves different phases that include designing, coding, testing, documentation and deployment. These tasks were traditionally performed by the employees in an organization, but software crowdsourcing assigns these tasks to crowd. Software crowdsourcing involves various types of tasks to be performed in different phases of software development; these tasks can be generalized in three broad categories:

1. Routine tasks: Involves tasks such as data collection, requirement gathering, minor coding, documentation, preliminary testing etc. Some of these tasks can be automated using artificial intelligence (AI) and machine learning.

2. Creative/Innovative tasks: Involves tasks such as writing new algorithms, creating new technical designs and research and development. These tasks involve highly skilled and innovative individuals.

3. Complex tasks: Involves huge and complex tasks that are divided into subtasks and performed by crowd, these subtasks are then combined to create the final solution. These may include complex coding, regression and acceptance testing, detail design documents etc.

For these different types of tasks software crowdsourcing implements different models and approach to solve these tasks. Some of these approaches have been listed below [4]:

\subsubsection{Knowledge discovery and management}

This approach is appropriate for the routine crowdsourcing tasks. This approach is ideal for information gathering, organization, reporting, translation, conversion, and other such tasks. The crowd is tasked to perform these tasks over the information and data spread across the organization. TxtEagle is an example of this approach which has been stated earlier. Another such example is the Peer-to-Patent Community Patent Review project [16]. Under a pilot project from 2007-09, the U.S. Patent and Trademark Office (USPTO) siphoned off a small number of patent applications it received to an online community. This community reviewed these applications for any evidence indicating that a similar invention already exists that would negate the originality of a patent application. These findings were then routed back to the USPTO.

\subsubsection{Broadcast Search}

Model suited for creative and innovative tasks. Here a task can be posted before the crowd and solutions are offered by individual workers. These solutions can then be reviewed by other members to select the best options. The Goldcorp challenge stated earlier is an example for this approach. Another such approach is adopted by InnoCentive.

\subsubsection{Distributed human intelligence}

This approach is suitable for complex tasks where crowd needs to collaborate to perform a complex task. Crowd needs to be organized and hierarchies are needed to be maintained. The concept is similar to other large scale distributed projects such as SETI@home and Rosetta@home [4]. The large problem is broken down into smaller problems requiring human intelligence and each individual in the crowd contribute for the processing. Notable example under this approach is Amazon Mechanical Turk where crowd accomplish a series of human intelligence tasks offered by the requester over the Mturk platform.

\subsection{Illustrations of Crowdsourcing Initiatives}

Several crowdsourcing initiatives have started in recent years where companies post a problem online and vast individuals contribute to offer solution and are suitably rewarded. These initiatives have not only been used for commercial purpose but also to champion the social causes. Initiatives like OpenStreetMap, ReCaptcha, Ushahidi, Peer Water Exchange etc. rely on volunteers whereas initiatives like InnoCentive, MTurk pay bounties to individuals for their contributions. To understand the workings of crowdsourcing, we will examine some of the most successful and profitable cases in a variety of industries.

\subsubsection{Ushahidi}

A mapping tool for crowdsourcing crisis information [5], Ushahidi enables public to communicate crisis information via SMS, e-mail, or web entry on real time basis. The information is time stamped and geo tagged to create a crisis map to report incidents to multiple organizations engaged in crisis response. The benefits range from timely crisis response from relief teams and policing agencies to efficient information flow [8]. Ushahidi has been used to map post-election violence in Kenya [5], anti-immigrant violence in South Africa [6], and manage relief efforts in Haiti and Chile [7].

\subsubsection{Threadless}

Threadless.com is a web-based t-shirt company that crowdsourced its design process for shirts through an online competition. Anyone may join Threadless community and has access to vote on designs or to submit them. Submitted designs are scored on a zero-to-five scale; designs are voted and the highest scoring designs are selected by Threadless to be printed. Winner receives US $\$ 1,500$ in cash and US $\$ 500$ worth of Threadless t-shirts and gift certificate [9].

\subsubsection{InnoCentive}

InnoCentive aims at tapping the talents of a global scientific community from innovative solutions to tough $R \& D$ problems through crowdsourcing. Scientists receive recognition and financial award for solving challenges. In one of such initiatives, a Canadian gold mining company Goldcorp launched "Goldcorp Challenge" in March 2000 offering more than $\$ 500,000$ in prize to 25 finalists who were required to examine the geologic data and identify the potential targets where next 6 million ounce of gold could be found in Ontario. More than 1400 participants from 51 countries registered for the challenge and identified 110 suspected and new deposits for gold [10] [15].

\section{IT OUTSOURCING: The Picture}

Technology outsourcing refers to outsourcing all or part of IT functions of an organization to an external party. Started in 1980s outsourcing is part of every organization in some or other form today, ranging from single task to complex tasks. Outsourcing has changed the structure of whole industries. The major motivation behind outsourcing is lack of resources 
and cost reduction. Countries like India, Philippines and China have become the destinations for outsourcing owning to the availability of cheap technical workforce. Over the decades outsourcing has developed toward stagnation as all organizations across globe today is embracing outsourcing for handling its IT operation. Therefore technology service providers are looking for options that would help them further reduce the cost and bring more profits for their clients. This has led to a fierce competition among these service providers to minimize the cost and maximize the productivity and bring about innovation. With advent of crowdsourcing the search seem to be finally over. Software crowdsourcing will offers a true output-based pricing; the workers are employed on a task basis and collaboration of huge workforce drive innovation. Later sections propose adoption of crowdsourcing in enterprises alongside outsourcing to harness the maximum benefits.

\section{ENTERPRISE CROWDSOURCING}

For organizations like IBM, TCS, Accenture etc. that cater top global enterprises, there is a need of more structured, secure and organized crowdsourcing services. This need has given rise to "Enterprise Crowdsourcing" which unlike general form of crowdsourcing implement different delivery model to inject the power and agility of crowdsourcing in routine business process to drive innovation, new business and save money.

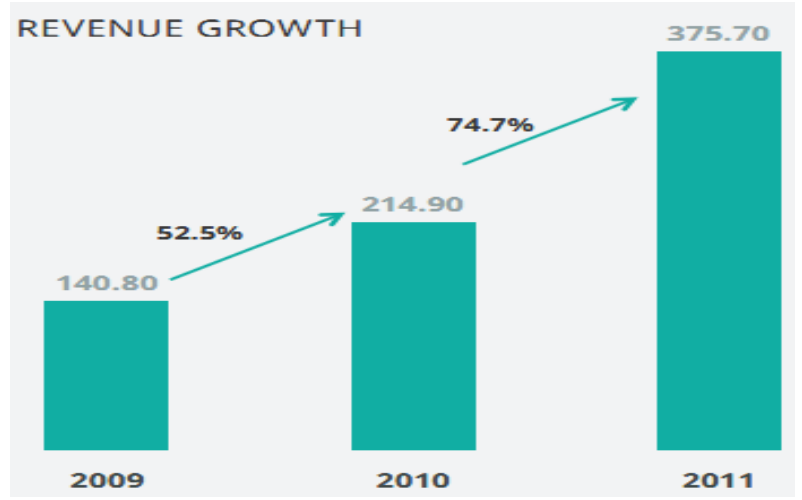

Fig 2: Revenue Growth of Crowdsourcing industry [14]

With online population approaching 3 billion by 2016 and projected to reach 5 billion by 2020, a new workforce has emerged that service providers can now harness [14]. The growth of global enterprise crowdsourcing is accelerating at a rapid pace. The above graph by Crowdsourcing.org shows the growth of 15 leading crowd services providers with revenues increasing from 140.80 million US\$ in 2009 to 375.70 million US\$ in 2011, an increase of more than $200 \%$ in just two years. Enterprise crowdsourcing tend to outsource the tasks to a crowd by implementing a customized delivery model that ensures best quality, offers maximum security, enhance productivity and optimize the cost of operations. Crowdsourcing can benefit all the business verticals whether it is banking and Finance, Telecommunication, Services, Healthcare, Energies or any other.

\subsection{Benefits of Enterprise crowdsourcing}

Crowdsourcing offers numerous benefits to organizations, some of these have been listed below:

1. Cost Saving - Minimizing the project cost gives a leading edge in today's highly competitive service based industry. Low operation cost provides greater business opportunities. Crowdsourcing has a productivity based pricing model that ensures payments being made for work and not for employees. The crowd workforce is relatively cheaper on account of low wages, no facility or infrastructure cost, no training cost.

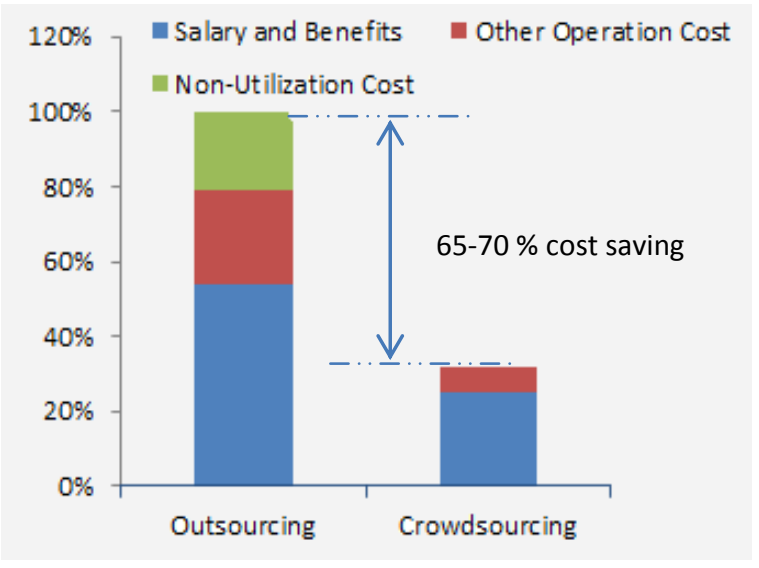

Fig 3: Net operating cost assessment for Crowdsourcing and Outsourcing [17]

The above figure depicts difference in the operational cost between traditional outsourcing and crowdsourcing. There is roughly $65-70 \%$ cost saving for organizations as crowdsourcing reduce the need of full-time employees, on demand workforce availability and payments can be disbursed to workers on per-task basis.

2. Productivity - Crowdsourcing can also greatly enhance productivity. As the software development cycle is highly modular, each phase of development is subdivided into several sub-tasks. Crowd workers can pick any of these subtasks. This imparts parallel processing of the entire process. In addition to this geographically distributed nature of crowd provide flexibility and ensures $24 X 7$ availability and throughput.

3. Time to Live - As stated earlier, $24 \mathrm{X} 7$ availability of crowd reduces the time for projects to go live in production, this in-turn reduce the cost of entire development lifecycle. Also this will help organizations to respond any crisis situation more efficiently and in a timely manner.

4. Scalability - Crowdsourcing provides an on-demand availability of skilled and specialized resources in any geographical location. This can greatly reduce reliability of a service provider on its bench workforce for any urgent situation. This will further reduce the cost of operation for these organizations and reduce the requirement of full time employees.

5. Innovation - Crowdsourcing will propel innovation as more ideas appear with increased collaboration of crowd workers this will help value creation and drive new business for these organizations.

\subsection{Challenges with Enterprise Crowdsourcing}

Although crowdsourcing offers several benefits to an organization but there are various challenges that are faced while implementing it. This section lists some major challenges:

1. Designing of Tasks - Software crowdsourcing will require tasks in each phase of development to be decomposed to large number of subtasks that will be performed by the crowd. It is a big challenge to design 
tasks that can be decomposed to smaller modules and then be put back together once completed with minimal manual intervention.

2. Security - is another major challenge for crowdsourcing, as all the tasks and communications are performed over internet crowdsourcing is constantly under threat which may include data breach, service hijack, SQL injections, malware, malicious insider etc.

3. Planning - As the complexity of the crowdsourced tasks is huge, planning and scheduling of the task is very critical to ensure the timely accomplishment of the objectives and enhance the productivity.

4. Quality assurance - This is a very important aspect for an organization's software development project; Organizations can design their own set of crowdsourced software development standards that shall be met by the developers. Also practices like peer-review and introduction of hierarchies in crowd can increase collaboration, help conflict resolution and improve quality standards.

5. Real-time collaboration and co-ordination - Timely completion of the sub-tasks and their collaboration can only be achieved through real-time collaboration and synchronization between the crowd-workers. This will also ease sharing of key resources among the crowd workers and avoid any stalemate.

6. Talent Pool - Creation of a highly skilled and talented crowd pool and its retention is a major challenge. Moreover owing to the diversity of crowdsourcing tasks different community of crowd workers is needed that can handle particular kind of tasks.

7. Rewards and remuneration - This is the primary motivation of crowd, therefore transparent payment systems and attractive remunerations are required to attract and retain the skilled crowd.

\section{CREATING AN ENTERPRISE CROWD PLATFORM}

This section proposes a framework for crowdsourced software development platform. This model aims to harness maximum benefits from software crowdsourcing and minimizing the challenges faced in implementing it. This section firstly propose process for software crowdsourcing followed by designing a crowdsourcing model followed by implementation techniques aimed at minimizing the above mentioned challenges.

\subsection{Process for implementing Software Crowdsourcing}

For implementing an enterprise software crowdsourcing initiative there are four basic steps [14] -

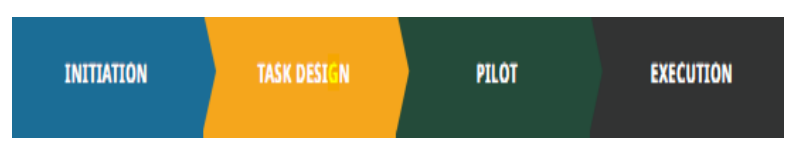

Step1: Initiation - Involves identification of projects that can be crowdsourced and outlining the benefits it can provide.

Step2: Task Design - Involves redesigning processes and workflows to drive software crowdsourcing effectively. Also involves breaking down of complex tasks to smaller tasks that can be assigned to different workers.
Step3: Pilot - Involves implementation of software crowdsourcing including development, testing, quality assessment, review of results, monitoring work and workers and rolling out plans of implementation.

Step4: Execution - Involves deployment of a full-fledged crowdsourcing system, scale the solutions and processes in production, monitor the product and provide production support.

The software crowdsourcing model presented in this paper allows the technology service providers to adopt crowdsourced software development with minimal modification to their current business model. Below diagram shows how crowdsourcing can be adopted along with outsourcing in current delivery model of service providers.

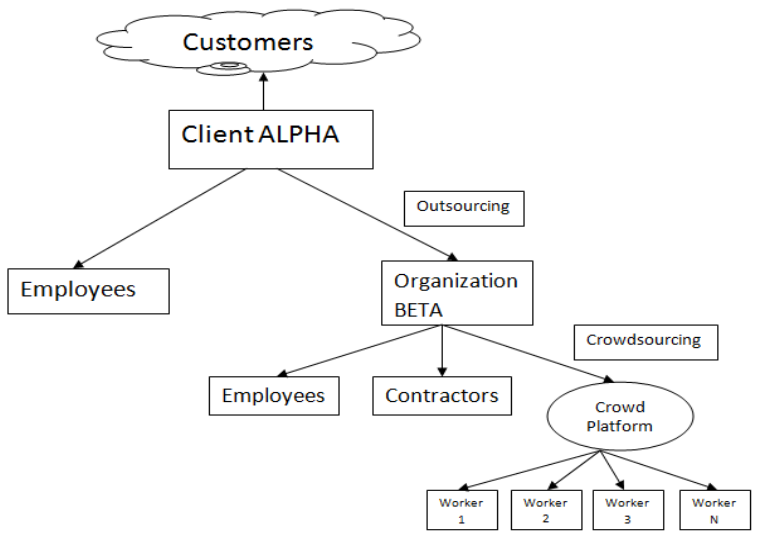

Fig 3: Business model with enterprise crowdsourcing

At presently, when a company, for example "Alpha" outsources its technology services to a service provider organization "Beta", the services are delivered to Alpha by employees or contractors at Beta. With development of a Crowd Platform at Beta the crowd workers can participate in delivering these services to Alpha. Software crowdsourcing will enable collaboration of crowd over a platform where they can participate in software development cycle right from development to deployment and can be suitably rewarded for tasks performed. As stated earlier, a crowdsourced system basically involves three elements [3]: organizations, workers and a platform for the crowd to collaborate. Apart from these elements a crowd system may also include other elements such as Artificial Intelligence tools, Integration tools, Automation Tools, Workflow design and management tools etc. Creation of an enterprise crowd platform require thorough planning; there are certain things that need to be considered before implementation such as -

1. Why crowdsourcing is needed and what benefits crowd workers brings to the organization that its employee cannot [8].

2. Identification of roles and responsibilities of crowd workers and extent to which they play role in the organization.

3. Identifying tasks that can be crowdsourced and designing of new workflows.

4. How can crowd collaborate and what model shall best suit for software development?

5. Determining the characteristics and qualification required for crowd, diversity of the crowd, rewards policy, 
retention of talent strategy and other such aspects of crowd.

After considering above listed points a crowd platform can be designed followed by an implementation strategy.

\subsection{Designing a Crowd Platform}

This section proposes design of a crowd platform that will help delivering software products through crowdsourcing; this will also elaborate workflows, processes and stages of development. Unlike a general crowdsourced system, this platform will employ private crowd and all the tasks will be crowdsourced by organization "Beta" to the crowd. Although the tasks being crowd sourced will actually be the tasks presently outsourced to organization "Beta" by its clients. The figure below highlights the stages involved in crowdsourced software development.

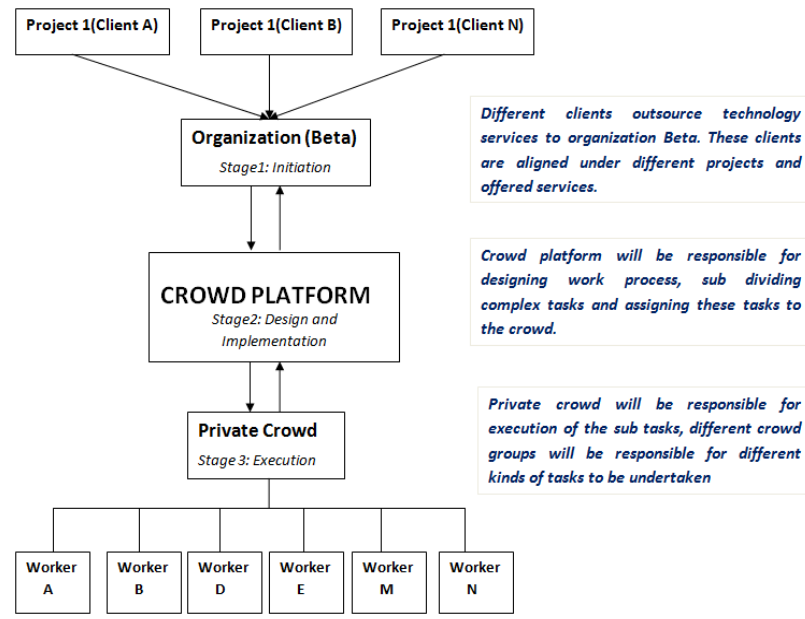

Fig 4: Stages involved in software crowdsourcing.

Stage 1: Initiation - In this stage a project "Alpha" for client Alpha raises a request for crowdsourcing its software development tasks with organization Beta's Crowd Platform. This platform will be managed as a separate project itself comprising of a team - service management, delivery management, technology architects, subject matter experts (SME), etc. The delivery management team at project "Alpha" identifies the business and technical requirements as specified by the client Alpha, highlight the priorities, lay down the guidelines for SLA and other such requirements with the Crowd Platform Service Management team at Beta.

Stage 2: Design and Implementation - After the crowd platform service management team gathers functional and technical requirements, the task for implementation of software crowdsourcing takes place. For more reliable, secure and cost effective implementation of crowdsourcing this paper emphasize on usage of cloud services in software crowdsourcing. The implementation steps has been listed below -

1. Creation of high level functional design by business analysts that highlights work flow and tasks to be undertaken.

2. Creation of technical design by SMEs and technical architects, this will be followed by designing of tasks and their decomposition into subtasks. These subtasks can then be hosted over the online platform for the crowd to pick them up.
3. As development being the first phase for development, subtasks hosted for development tasks can be undertaken by the crowd workers. The workers can choose the tasks based on their technical skills, all the tools required for development will be offered over the private cloud hosted by organization Beta as SaaS (Software as a Service).

4. After a subtask is completed it is submitted by the crowd worker over the platform. The completed task is assessed by SME at Beta; if the result is satisfactory the payment is made to the crowd worker and the subtask is sent for integration with other successfully completed subtasks. After all the subtasks have been completed they are integrated together into an end product.

5. After integration of the subtasks end results gives the solution for the complex task. This end product undergoes quality analysis by technical architect at Beta.

6. After quality analysis signoff is provided for the developed product in stage 5, following which testing starts. Testing covers all subtasks as well as the complex tasks. This includes various forms of testing such as unit testing, functional testing, system testing, integration testing, performance testing, and acceptance testing. All the tools and resources required for testing are offered as SaaS or PaaS or IaaS over cloud.

7. After testing results are verified by SMEs payments are made to the crowd workers involved with testing. The final software product will meanwhile be sent for review to the crowd platform service management team and product delivery team at project Alpha for business review.

8. Once the nod is provided by the delivery team at Alpha the product is prepared for implementation to production else the product is sent back for changes. The changes are then made by the crowd workers and the above processes repeat. Before implementation into production the documentation part can also be crowdsourced.

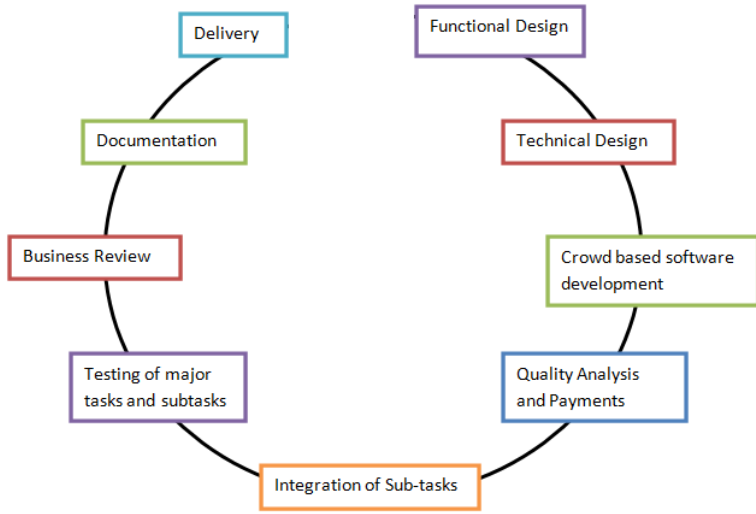

Fig 5: Steps involved in implementation of crowdsourcing.

Stage 3: Execution - After the product has been delivered to the project team at Alpha it can undergo further acceptance testing. Finally, this crowdsourced software product can be implemented into production by the delivery team at project Alpha on behalf of organization Beta like any other services delivered by Beta. 


\subsection{Implementing the Crowd Platform}

This section discusses strategies for implementation of a strong crowd platform which will help in overcoming present challenges. The implementation is based on service oriented architecture.

Creating a Crowd Platform: Any crowd initiative requires a platform that enables its crowd workers to collaborate and share their efforts. To build secure, scalable, and cost effective platform organizations shall use private cloud services. Cloud will offer SaaS, PaaS and IaaS services which will be needed during different phases of software development lifecycle. Using cloud will greatly reduce the cost as well as efforts of implementation of the crowd platform. A crowd worker can login into to the platform through a portal and after his or her authentication the workers can commence work over the task chosen by them using the tools offered, communicate and share data in real time using communicators such as Microsoft Lync.

Designing of tasks: When any project wants to crowdsource its software development assignment, a request shall be raised with the crowd platform service management team in organization Beta. After requirement specification a technical design is created based on which the software development task is created. This task is then divided into sub modules/tasks for development. These sub modules/tasks are then further divided into micro-tasks. These micro-tasks are posted over the crowd portal where these tasks can be picked by crowd workers from respective teams. As number of micro-tasks is huge Artificial Intelligence tools can be developed and deployed to designing tasks, dividing tasks and integration of subtasks on completion. This will minimize the plausibility of errors due to manual intervention.

Security: As the entire software development with crowdsourcing occurs over internet it is extremely important to ensure security and safety. Firstly organizations shall implement a "private" crowd that allows identification of crowd workers and helps building trust on the workers. Other techniques are also implemented to ensure the security and safety. Some of the measures include - data compliance standards such as SSAE16, ISO/IEC27017, encryption of data, multi-factor authentication, digital signatures etc. for data security; identity and access management, audits and logging, user authorizations etc. to avoid service hijacking and network intrusion.

Quality Assurance: Quality assurance is an important aspect of software crowdsourcing that ensure delivery of quality services to the clients. There shall be a matrix for quality assurance, with technical SMEs at first level followed by crowd service management team and finally the client delivery teams that reviews the software product for quality. In addition, standards coding practices can be established, code templates can be provided for the workers etc. To ensure the quality of workers formal and informal tests can be administered periodically. This would ensure both quality and integrity.

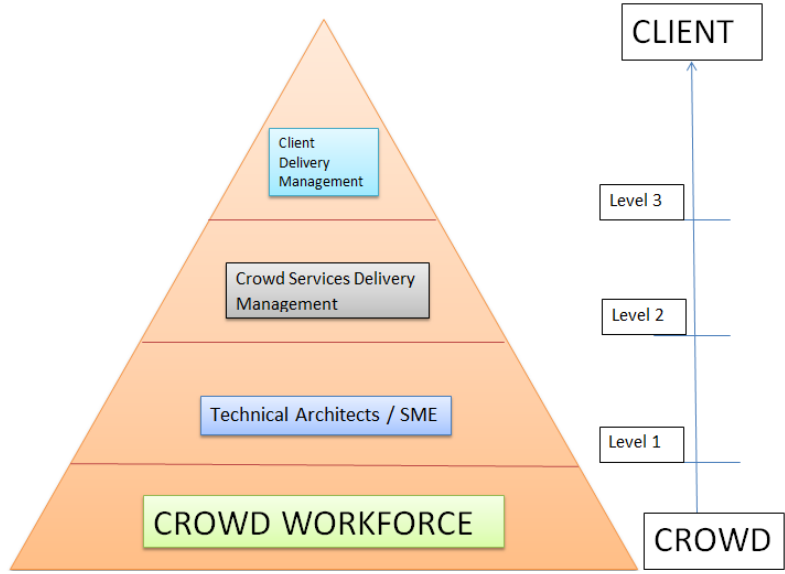

Fig 6: Quality assurance matrix on Crowd Platform.

Recruiting crowd workers: Delivering high performance will require best workers in the crowd. As the crowdsourced tasks are diverse both in terms of creativity and technicality crowd workers with different technical and creative skills are needed. But the question arises as how to acquire motivated and talented crowd workers? The best solution is tie ups with various technical institutions. The students at these institutions are highly motivated as working with these organizations will help them improve their technical skills and offer work experience. This will offer a seamless pool of talented and motivated workers. Workers can also be recruited online by testing their skills through online test and assignments.

Retaining crowd workers: Another major challenge is how to retain the quality crowd workers? As monetary benefits are primary motivation for the crowd workers, lucrative incentives can be offered to crowd workers. Also a trust has to be built up between the organization and the crowd workers by creating a fair payment system. Bonuses can be offered to the workers depending upon their skills and talent which will be rated periodically.

\section{CONCLUSION}

This paper provided an insight into software crowdsourcing. Today global outsourcing market is more than 300 billion USD and the biggest employer in IT industry but few decades ago it faced reluctance when this initiative started. Crowdsourcing is a radical technology and in its early stages of development, with advancement in technology coupled with research it shall become global technology trend. Crowdsourcing offers numerous benefits service companies like Accenture such as faster time to live, availability of scalable and flexible work force, increased productivity and huge cost savings. Above all crowdsourcing will give a leading edge to service providers in the highly competitive technology service industry. This paper has also proposed a software crowdsourcing model for service providers outlining the designs and implementation strategies. With further research and development software crowdsourcing can be render great services and benefits alongside outsourcing for the organizations.

\section{REFERENCE}

[1] Howe, J. (2006f) 'The Rise of Crowdsourcing', Wired, 14(6).At:

http://www.wired.com/wired/archive/14.06/crowds.html

[2] Enrique Estellés-Arolas, Fernando González-Ladrón-deGuevara (2012), 'Towards an integrated crowdsourcing definition', Journal of Information Science. 
[3] Eric SCHENK, Claude GUITTARD (December 7, 2009), Crowdsourcing: What can be Outsourced to the Crowd, and Why? Halshs-00439256, version 1 - 8 Dec 2009.

[4] Daren C. Brabham (2011), Crowdsourcing: A Model for Leveraging Online Communities .

[5] Greenough, P., Chan, J., Meier, P., Bateman, L., \& Datta, S. (2009). Applied Technologies in Humanitarian Assistance: Report of the 2009 Applied. Prehospital and Disaster Medicine , 206-209.

[6] Fildes, J. (2010, March 1). Net puts Kenya at centre of Chile rescue efforts. http://news.bbc.co.uk/1/hi/world/americas/8543671.stm .

[7] Ramirez, J. (2010, March 03). Ushahidi technology saves lives in Haiti and Chile. Retrieved from Newsweek: http://blog.newsweek.com/blogs/techtonicshifts/archive/ 2010/03/03/ushahidi-technology-saves-lives-in-haiti-andchile.aspx

[8] Ankit Sharma (2010), Crowdsourcing Critical Success Factor Model, Strategies to harness the collective intelligence of the crowd

[9] Daren C. Brabham (2008), Crowdsourcing as a Model for Problem Solving, An Introduction and Cases, Convergence: The International Journal of Research into New Media Technologies.
[10] InnoCentive, Harnessing the Global Talent Pool to Accelerate Innovation, EXECUTIVE WHITE PAPER.

[11] Andy Bethurum(September, 2012), Crowd Computing: An Overview, CrowdComputing Systems, Inc.

[12] Jafar Muhammadi and Hamid R. Rabiee,Department of Computer Engineering, Sharif University of Technology, Tehran, Iran. Crowd computing: a survey. arXiv:1301.2774v1 [cs.AI] 13 Jan 2013.

[13] Braden Kelley, The Crowd Computing Revolution.

[14] massolution (january 2013), The Crowd in the Cloud: Exploring the Future of Outsourcing.

[15] Goldcorp Challenge Winners! (2001) The Goldcorp Challenge (12 March), URL (accessed May 4, 2007): At: http://www.goldcorpchallenge.com/challenge1/winnersli st/chaleng2.pdf.

[16] Beth Simone Noveck, 2006. "Peer to Patent": Collective Intelligence, Open Review, and Patent Reform," Harvard Journal of Law \& Technology, volume 20, number 1, pp. 123-262.

[17] "Every Crowd has a silver lining", Eric Simon (2011), Everest Global Inc. 